\title{
Mild Ischemic Mitral Regurgitation: Is Revascularization Enough for Every Patient?
}

\author{
Hunbo Shim, MD, ${ }^{1}$ Ji-won Hwang, MD,${ }^{2}$ Won-Sang Chung, MD, ${ }^{1}$ Chun Ki Kim, MD,${ }^{3}$ Byung Jo Park, MD, \\ Young Tak Lee, $\mathrm{MD},{ }^{4}$ Wook Sung Kim, $\mathrm{MD}^{4}$ \\ ${ }^{1}$ Department of Thoracic and Cardiovascular Surgery, Hanyang University College of Medicine, Seoul, Korea; ${ }^{2}$ Division of \\ Cardiology, Department of Medicine, Ilsan Paik Hospital, Inje University School of Medicine, Kyunggi-do, Korea; ${ }^{3}$ Department of \\ Medicine, Hanyang University College of Medicine, Seoul, Korea; ${ }^{4}$ Department of Thoracic and Cardiovascular Surgery, Samsung \\ Medical Center, Sungkyunkwan University School of Medicine, Seoul, Korea
}

\section{ABSTRACT}

Background: The progress of mild ischemic mitral regurgitation (MR) after isolated coronary artery bypass is not clear. We aimed to determine the proportion of patients with mild ischemic MR undergoing isolated coronary artery bypass grafting (CABG) presenting with regression of or persistent MR one year after CABG and to identify the significantly different echocardiographic variables between regressing and persistent MR.

Methods: Sixty-three patients with preoperative mild ischemic MR were categorized into MRregression or MR-persistence group one year after isolated CABG. The echocardiographic indices, indicating mitral leaflet configuration and remodeling of the left ventricle (LV), were measured before and one year after the surgery.

Results: One year after CABG, MR regressed in $60 \%$ (38/63) and persisted in $40 \%(25 / 63)$ of the patients. The left ventricular diameter, volume, and sphericity and anteroposterior diameter of the mitral annulus improved only in the MR-regression group, while the ejection fraction improved in both groups $(47.7 \% \pm 12.4 \%$ from $40.1 \% \pm 11.3 \%$, $P<.001$ in the regression group and $43.2 \% \pm 14.0 \%$ from $39.3 \% \pm 11.6 \%, P=.035$ in the persistence group). A $>15 \%$ decrease in the LV end-systolic volume was noted more frequently in the MR-regression group $(60.5 \%$ versus $30 \%$, $P=.027)$. The leaflet angle did not show asymmetry or significant changes in both groups.

Conclusions: Isolated CABG improved mild MR in most patients with mild ischemic MR. These patients showed greater reverse remodeling after revascularization than the patients with persistent MR after isolated CABG. Additional tests, which can predict $L V$ reverse remodeling, are needed to predict persistent MR.

Received October 6, 2019; received in revised form April 15, 2020; accepted April 20, 2020.

Correspondence: Wook Sung Kim, MD, PhD, Department of Thoracic and Cardiovascular Surgery, Samsung Medical Center, Sungkyunkwan University School of Medicine, 81 Irwon-ro, Gangnam-gu, Seoul 06351, Korea; + 82-23410-3486; fax: +82-2-3410-0089 (e-mail: wooksung.kim@samsung.com).

\section{INTRODUCTION}

A mild degree of ischemic mitral regurgitation (MR) often is considered a minor issue during surgical planning, despite concern for its progression to heart failure. Heart failure from ischemic MR increases mortality, even when mild [Aronson 2006; Lehmann 1992; Feinberg 2000; Grigioni 2001]. Currently, the most plausible mechanism for ischemic MR is tethering of the mitral valve resulting from local or global ventricular wall motion abnormalities, due to previous myocardial infarction. However, this tethering is not the sole problem related to the underlying mechanism, due to the complex interplay of various vectors influenced by left ventricle (LV) function, dilatation, and remodeling. Although the tethering and tenting of the mitral leaflet is the final common pathway mediating leaflet malcoaptation and incomplete closure, patients with coronary artery disease, especially those referred for surgery, present with a wide spectrum of pathologic changes in the LV, annulus, and mitral leaflets. In this context, completely abolishing the MR is difficult using the simplified common strategy. Hence, differentiating patients is important to maximize the effectiveness of surgical treatment.
(A)

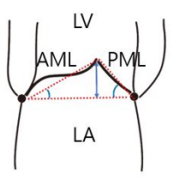

(C)

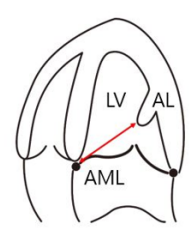

(B)

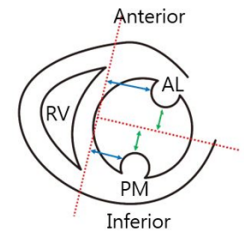

(D)

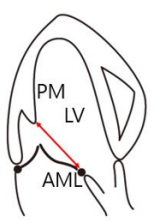

A. Mitral leaflet configuration. B. Lateral and posterior displacement of each papillary muscle, interpapillary distance. C. Apical displacement of anterolateral papillary muscle. D. Apical displacement of posterolateral papillary muscle. $\mathrm{AL}=$ anterolateral papillary muscle; $\mathrm{PM}=$ posteromedial papillary muscle; $\mathrm{AML}=$ anterior mitral leaflet; $\mathrm{PML}=$ posterior mitral leaflet; $L V=$ left ventricle; $R V=$ right ventricle 
Table 1. Preoperative demographic, clinical, and operative data

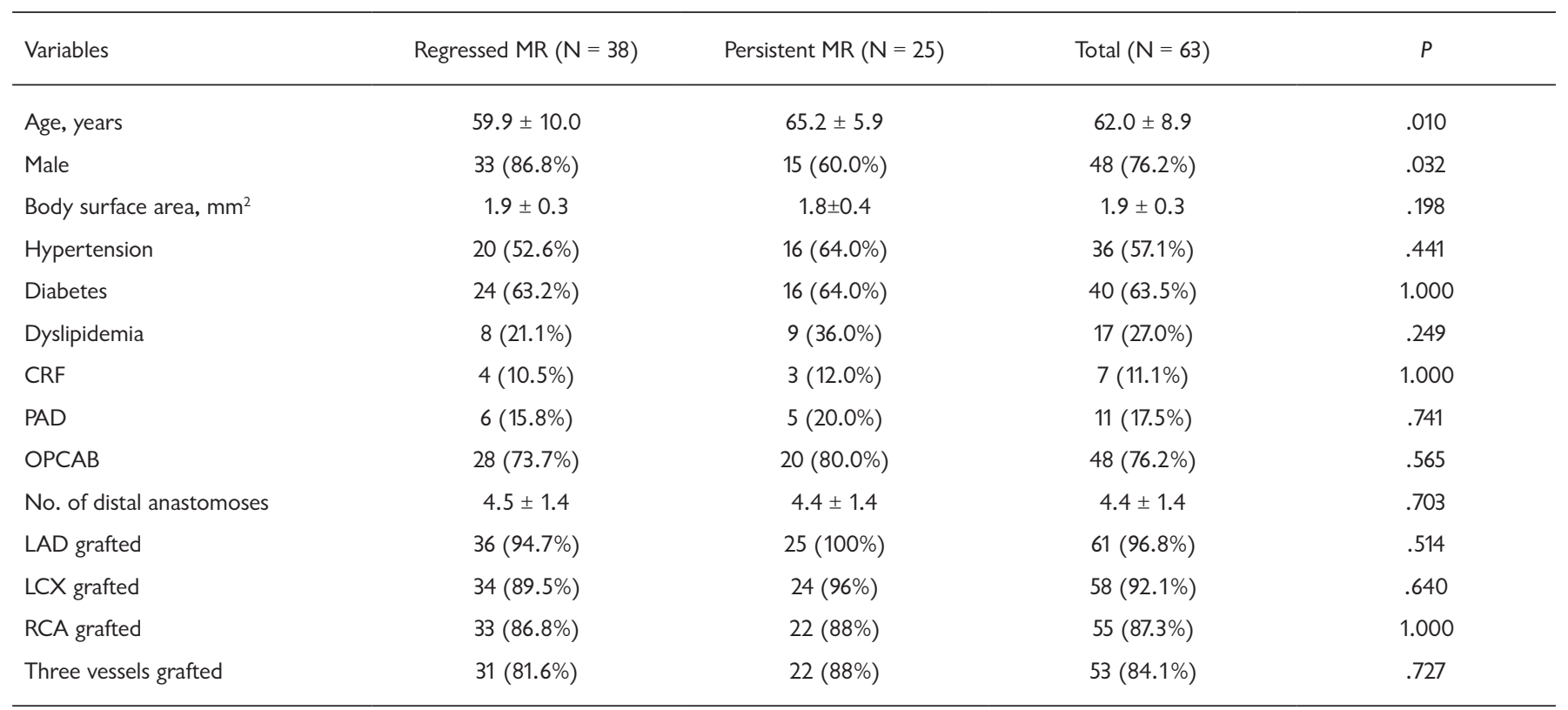

$C R F=$ chronic renal failure; $L A D=$ left anterior descending artery; $L C X=$ left circumflex artery; OPCAB = off-pump coronary artery bypass; $P A D=$ peripheral artery disease; $\mathrm{RCA}=$ right coronary artery

Unfortunately, the current guidelines for ischemic MR do not reflect this complexity; this ambiguity led us to conduct the present study. The aim of our study was: 1 ) to determine what percentage of patients with mild ischemic MR undergoing isolated coronary artery bypass grafting (CABG) show regression of $M R$ versus persistent $M R$ one year after $C A B G$, and 2) to find variables that significantly differ between an MR-regression and an MR-persistence patient group, which might provide additional clues to understand the mechanisms underlying ischemic MR.

\section{PATIENTS AND METHODS}

Study subjects: The records of 63 patients with mild ischemic MR who underwent isolated CABG between January 2007 and December 2017 were reviewed. Eligible patients had a documented history of myocardial infarction more than 16 days before their baseline assessment and/or an electrocardiogram (ECG) with a significant Q-wave. Patients were excluded from the study if they had a history of previous heart surgery, a non-sinus rhythm at follow-up, organic abnormalities in the mitral leaflet, or other associated cardiac diseases other than tricuspid regurgitation. Patients without available echocardiography images allowing quantitative analysis of the intracardiac structures also were excluded. Lovastatin and carvedilol were administered postoperatively to all patients. Captopril was added for patients with ejection fractions (EFs) $\leq 40 \%$. The institutional review board approved this retrospective study and waived the need for written informed consent.
Echocardiographic measurements: Two-dimensional and Doppler transthoracic echocardiography were performed within seven days and one year after CABG. MR quantitatively was assessed by measuring the ratio of the MR color flow jet area to the left atrium area using color mapping of the apical four-chamber view during cardiac systole, graded as mild $(<20 \%)$, moderate $(20 \%-40 \%)$, or severe ( $>40 \%)$.

Mitral valve configuration: The mitral valve configuration was assessed in the apical four-chamber view during mid-systole. The coaptation height was defined as the distance from the coaptation point of the mitral leaflets to the annular plane, while the tenting area was measured as the area created by the mitral leaflets and annular plane. The anterior and posterior leaflet angles were the angles created by the annular plane and the line from the anterior and posterior leaflet hinge points to the coaptation point, respectively (Figure). The ratio of the anterior to posterior leaflet angles was calculated to measure symmetry: the closer the ratio to 1 , the more symmetric the tethering.

Local LV remodeling and function: LV volumes and EFs were calculated using the modified biplane Simpson method. The LV sphericity index was defined as the ratio of the longaxis length divided by the LV short-axis length in the fourchamber view, both during end-diastole and end-systole. LV mass was calculated from the septal and posterior wall thicknesses and the LV internal dimension at end-diastole and then divided by the body surface area to calculate the $\mathrm{LV}$ mass index. A reduction in LV end-systolic volume (LVESV) of $>15 \%$ at one year after discharge was used as a surrogate of LV reverse remodeling. 
Table 2. LV remodeling and sphericity

\begin{tabular}{|c|c|c|c|c|c|c|}
\hline $\operatorname{LVESD}(\mathrm{mm})$ & $43.7 \pm 6.8$ & $37.9 \pm 6.8$ & $<.001$ & $42.0 \pm 10.7$ & $42.5 \pm 13.0$ & .647 \\
\hline LVEDV $(\mathrm{mm})$ & $149.6 \pm 45.0$ & $125.1 \pm 43.2$ & .001 & $138.0 \pm 58.8$ & $136.7 \pm 60.5$ & .840 \\
\hline LVEF (\%) & $40.1 \pm 11.3$ & $47.7 \pm 12.4$ & $<.001$ & $39.3 \pm 11.6$ & $43.2 \pm 14.0$ & .035 \\
\hline $\operatorname{LVMI}\left(\mathrm{g} / \mathrm{m}^{2}\right)$ & $129.2 \pm 24.1$ & $113.0 \pm 35.6$ & .006 & $112.4 \pm 32.4$ & $115.8 \pm 38.4$ & .644 \\
\hline Systolic LV sphericity & $1.8 \pm 0.3$ & $1.9 \pm 0.3$ & .108 & $1.7 \pm 0.3$ & $1.8 \pm 0.4$ & .074 \\
\hline Diastolic LV sphericity & $1.5 \pm 0.2$ & $1.6 \pm 0.2$ & .047 & $1.5 \pm 0.3$ & $1.6 \pm 0.2$ & .380 \\
\hline AP diameter $(\mathrm{mm})$ & $25.5 \pm 2.0$ & $23.7 \pm 1.6$ & $<.001$ & $25.1 \pm 2.3$ & $24.2 \pm 1.8$ & .055 \\
\hline
\end{tabular}

AP = Anteroposterior; LV = left ventricle; LVEDD = left ventricular end-diastolic diameter; LVEDV = left ventricular end-diastolic volume; LVEF = left ventricular ejection fraction; LVESD = left ventricular end-systolic diameter; LVESV = left ventricular end-systolic volume; Pre-OP = preoperative

Papillary muscle (PM) displacement: A line between the LV septal insertion points on the right ventricle and another right-angled line originating from the center of the first line were created as references. The lateral and inferior displacements of each papillary muscle were measured as the distances from these lines. The interpapillary muscle distance directly was measured. Apical displacement was measured, during mid-systole using the mitral anterior leaflet hinge point as a reference. The lengths from the anterolateral and posteromedial PM tips to the reference were measured in the apical four- and three-chamber views, respectively (Figure).

Statistical analyses: Shapiro-Wilk tests were used to assess the group-wise normality of the data distributions. Categorical variables are presented as frequencies and percentages, and continuous variables as means \pm standard deviation (SD). Differences between groups in postoperative MR were evaluated using chi-square tests for categorical variables. Differences in continuous variables between groups were assessed with independent $t$-tests. Paired sample $t$-tests were used to evaluate changes in echocardiographic parameters pre-operation and at one year after the operation. A $P$-value $<.05$ was considered statistically significant.

\section{RESULTS}

Patient characteristics: The patient characteristics at preoperative baseline are summarized in Table 1. (Table 1) Of the 63 patients, MR had regressed in 38 patients $(60.3 \%$, MRregression group) and persisted (30.2\%) or progressed (9.5\%) in 25 patients (39.7\%, MR-persistence group) at one year after surgery. There were no significant differences between the two groups except for age and sex. Age was lower and male sex was dominant in the MR-regression group. Patients mainly underwent off-pump CABG in both groups (76.2\%).
The left anterior descending artery was grafted in $96.8 \%$ of patients, while the left circumflex and right coronary arteries were grafted in $92.1 \%$ and $86.8 \%$ of patients, respectively. All three vessels were grafted in $84.1 \%$ of patients. The mean number of distal anastomoses was $4.4 \pm 1.4$, representing global ischemia.

Changes in LV geometry and function: There was significant reverse $L V$ remodeling in the MR-regression group, as illustrated by decreases in diameter, volume, AP diameter, and mass as well as improvements in LV systolic function at one year. LV sphericity was improved only during diastole even in this group. In contrast, patients in the MRpersistence group did not show any improvements in LV geometry, despite significantly improved LV systolic function (Table 2). A 15\% reduction in LVESV at one year of follow up occurred in $60.5 \%$ of patients in the MR-regression group and in $32 \%$ of patients in the MR-persistence group $(P=.027)$.

PM displacement: The posterior displacement of the posteromedial PM decreased significantly $(P=.005)$ with decreased interpapillary muscle distance $(P=.024)$ in the MR-regression group. In the MR-persistence group, the posterior displacement of the anterolateral PM improved $(P=.035)$. The interpapillary muscle distance also decreased in this group $(P=.012)$. The apical displacement of each PM did not change in either group (Table 3).

Mitral valve configuration: The leaflet configuration data are shown in Table 4. There was no specific finding in terms of leaflet configuration except for the tenting area. The tenting areas were reduced in both groups $(P<.001$ in both). The asymmetries were minimal and had not changed in either group at one year of follow up. The anterior and posterior leaflet angles were less than $25^{\circ}$ and less than $45^{\circ}$, respectively, and remained the same at follow up. The tenting height was less than $1 \mathrm{~cm}$ in both groups and did not change in either group. 
Table 3. Papillary muscle displacement

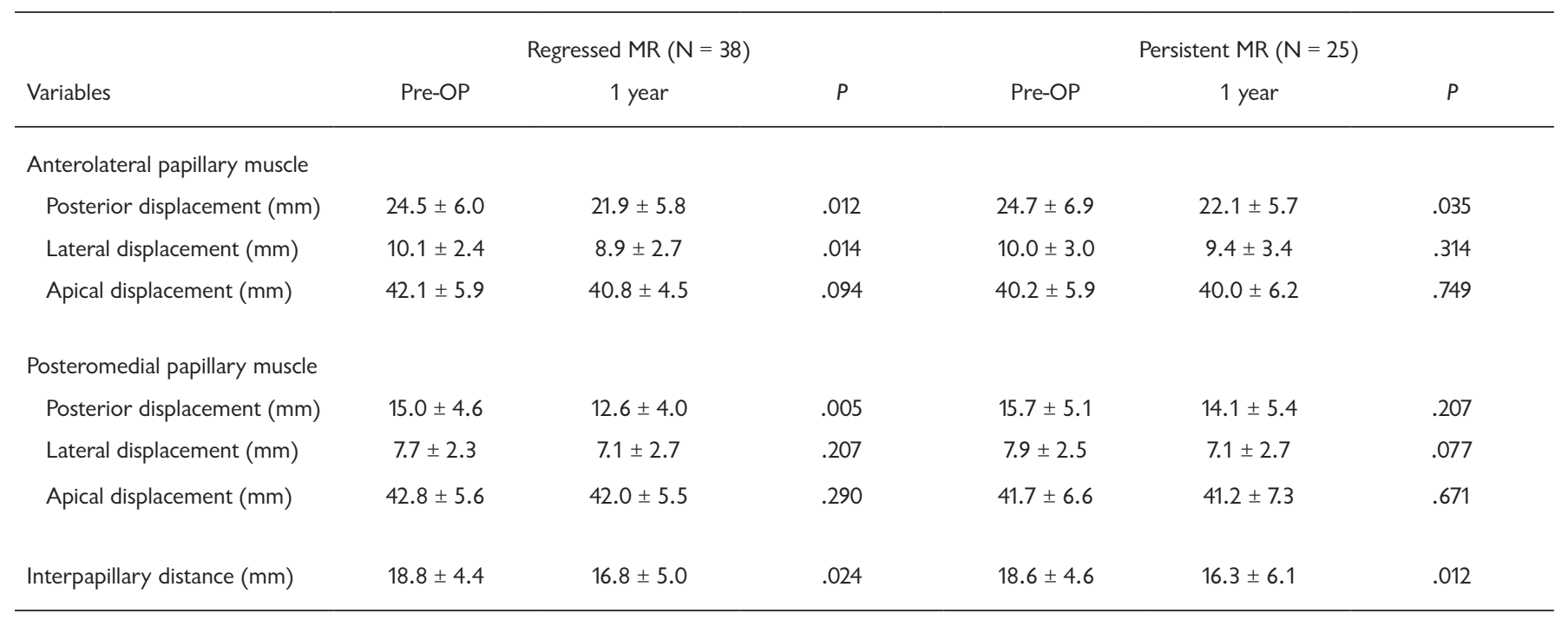

$\mathrm{MR}=$ mitral regurgitation; Pre-OP = preoperative

\section{DISCUSSION}

The findings of this study can be summarized as follows. Following isolated CABG, 1) regurgitation disappeared in the $60.3 \%$ of patients with mild ischemic MR, 2) the mitral leaflet angle had not changed one year after surgery in either group, and 3) all parameters indicating $L V$ remodeling improved in the patients with regressed MR, whereas most of these parameters did not improve in patients with persistent MR. Concomitant mitral valve surgery at the time of CABG is considered a high-risk cardiac surgery, and the current AHA/ ACC guideline recommends it for secondary MR in patients undergoing CABG only in cases with symptoms of severe MR [D'Agostino 2018; Nishimura 2017]. The approach to ischemic MR of moderate degree in patients undergoing CABG remains controversial [Michler 2016; Smith 2014]. Although CABG may be able to eliminate moderate MR in selected patients, if reverse LV remodeling by viable myocardium, which affects papillary muscle geometry, does not occur, MR seems to remain [Salmasi 2018; Ji 2019; Penicka 2009]. In this condition, a mild degree of MR often is overlooked when CABG is performed. However, even mild MR increases the risk for future heart failure and short- and long-term mortality [Aronson 2006; Lehmann 1992; Feinberg 2000; Grigioni 2001]. Although the underlying pathophysiology of ischemic $M R$ is presumably the same regardless of the degree of $M R$, the natural history of regression versus persistence of mild ischemic MR is not clearly known. We therefore need to investigate how many and which patients will have persistent MR after isolated CABG in order to implement a surgical strategy. Our study observed MR irreversibility in $40 \%$ of patients after revascularization. Generally, incomplete closure of the mitral valve arises from an imbalance between the increase in tethering force caused by LV remodeling and the decrease in closing force due to LV dysfunction after remote myocardial infarction [Agricola 2004; He 1997]. Our results are in line with this theory, showing significant reverse remodeling and improved ejection fraction in patients with regressed MR after isolated CABG, while LV remodeling parameters did not significantly change in patients with persistent $M R$, even though ejection fractions mildly improved. LVEDV and AP diameter indicating $L V$ dilatation were improved only in the MR-regression group. However, not only LV dilatation but also additional geometric changes in the mitral apparatus are required to create chord tension [Otsuji 2002]. The papillary muscle, especially the posteromedial papillary muscle, is displaced toward the posterior direction in ischemic cardiomyopathy, which is indicated by increased sphericity [Otsuji 1997; Kwan 2003]. Kono et al. found that sphericity was correlated with MR severity [Kono 1992; Sabbah 1993; Sabbah 1992]. These findings are concordant with our results, in that sphericity and posteromedial papillary muscle displacement improved only in patients without MR after isolated CABG.

The symmetry of the mitral leaflet is another important factor in the evaluation of ischemic MR, because asymmetry exaggerates MR severity irrespective of global $L V$ remodeling [Zeng 2014]. Our patients showed a symmetry of roughly 1.3, which is within the normal limits; this value did not change in either group after CABG. This finding also supports the notion that MR is caused by an imbalance in the competing forces between tethering from global LV dysfunction and closing by $\mathrm{LV}$ contractility rather than by unilateral leaflet tethering from focal LV dyskinesia. Given that our patients were referred for surgery because of multi-vessel disease and that an average of 4.5 target vessels were grafted, it is possible these patients had global LV lesions. In this regard, our study reflects the situation in real-world medical practice.

The overall results showing differences in LV remodeling parameters between the two groups indicate that the amount of viable myocardium was smaller in the MR-persistence group 
Table 4. Mitral leaflet configurations

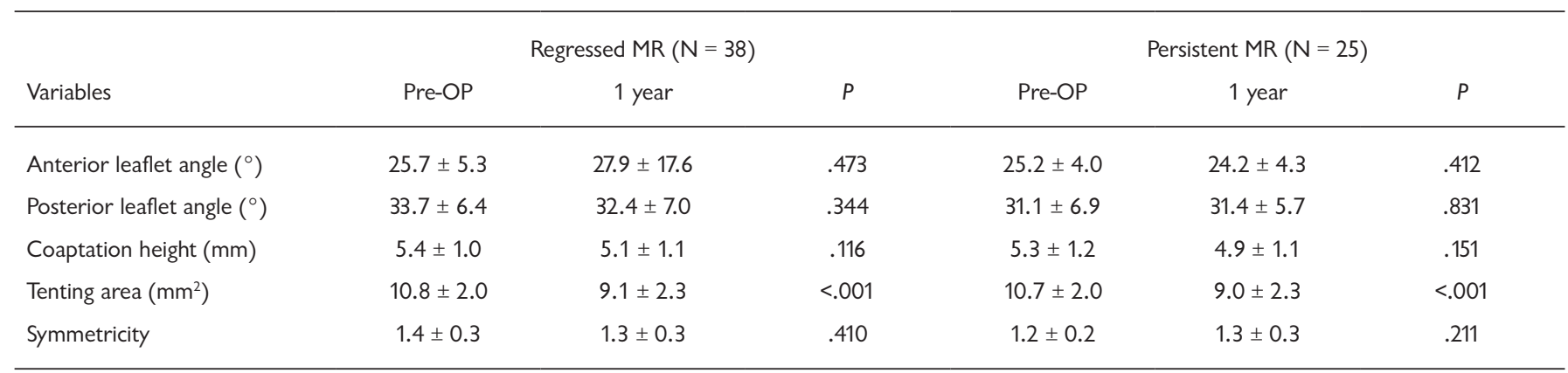

$\mathrm{MR}=$ mitral regurgitation; Pre-OP = preoperative

than in the MR-regression group. Therefore, performing viability tests before the operation may potentially be useful in determining if a patient with mild ischemic MR would benefit from concomitant MR repair rather than isolated CABG.

Prophylactic MR repair might be required for patients who are not expected to show reverse remodeling in preoperative viability tests. Lehmann et al. reported a nearly fourfold increase in the 1-year for patients with acute myocardial infarction and mild MR compared with that for the patients without MR, a result reproduced by Feinberg, who reported a hazard ratio of 2.31 (95\% confidence interval 1.03-5.20) for 1-year mortality [Lehmann 1992; Feinberg 2000]. Since only a part of the patient population underwent revascularization in their study, we expected better outcomes in our study, where all patients showed complete revascularization. However, MR still adversely affected mortality and should, thus, be eliminated. Annuloplasty with a ring remains the gold standard among several surgical options. However, the high failure rate of annuloplasty with ring in ischemic MR raises doubts on whether any patient group could be considered a candidate group for this technique [Michler 2016; Acker 2014; Goldstein 2016; McGee 2004]. Several studies have reported that echocardiography measures of increased tethering, such as anterior and posterior leaflet angles, predict recurrent MR. Magne et al suggested that a posterior leaflet angle of $\geq 45^{\circ}$ increases the probability of failed mitral repair [Magne 2007]. As annuloplasty shifts the coaptation zone more anteriorly, the posteromedial PM location can be further distorted and lie outside the annulus ring, and the tethering effect on the posterior leaflet makes it less likely to coaptate at the anteriorly shifted coaptation zone [Hung 2012]. Kuwahara et al. also reported an increased posterior leaflet angle after annuloplasty [Kuwahara 2006]. The posterior leaflet angle in our study was $31.1 \pm 6.9^{\circ}$, far smaller than the known predictor. Some authors have suggested an anterior leaflet angle of $25-39.5^{\circ}$ as an independent predictor for MR recurrence [Dal-Bianco 2009; Hsuan 2013; Meris 2012; Nesta 2003; Tibayan 2003] and the anterior leaflet angle in the present study, at $25.1^{\circ}$, was also in the lower limit of this range. Our patients were not at risk with respect to these aspects as most patients referred for surgery had three-vessel disease, which shows global LV remodeling, including that of the anterior wall infarct. Accordingly, we believe that concomitant annuloplasty may be performed in these patients without concern for tethering augmentation.

Limitations: This study has the inherent limitations of retrospective studies and a small sample size and presents a single-center experience. In addition, we were not able to establish a direct association between our results and preoperative viability test findings. Since the institutional strategy was to perform preoperative viability tests only for patients with $\mathrm{LV}$ ejection fractions of less than $30 \%$, we were unable to obtain data on these tests. Future studies will include preoperative viability tests for all cases of ischemic MR and will be able to report a direct association between the study findings and preoperative viability test results.

\section{CONCLUSION}

Regurgitation resolved by complete revascularization in $60 \%$ of the patients with mild ischemic MR. Compared to these $60 \%$, the remaining $40 \%$ with persistent MR did not show significant reverse remodeling or asymmetric leaflet tethering. In patients with mild ischemic MR, preoperative viability testing may be useful in sorting out candidates who may potentially benefit from prophylactic MR repair. Our findings suggest that annuloplasty should be performed in such patients without concern for augmentation of tethering.

\section{REFERENCES}

Acker MA, Parides MK, Perrault LP, et al. 2014. Mitral-valve repair versus replacement for severe ischemic mitral regurgitation. The New England journal of medicine. 370(1):23-32.

Agricola E, Oppizzi M, Maisano F, et al. 2004. Echocardiographic classification of chronic ischemic mitral regurgitation caused by restricted motion according to tethering pattern. European journal of echocardiography: the journal of the Working Group on Echocardiography of the European Society of Cardiology. 5(5):326-334.

Aronson D, Goldsher N, Zukermann R, et al. 2006. Ischemic mitral regurgitation and risk of heart failure after myocardial infarction. Archives of internal medicine. 166(21):2362-2368. 
D'Agostino RS, Jacobs JP, Badhwar V, et al. 2018. The Society of Thoracic Surgeons Adult Cardiac Surgery Database: 2018 Update on Outcomes and Quality. The Annals of thoracic surgery. 105(1):15-23.

Dal-Bianco JP, Aikawa E, Bischoff J, et al. 2009. Active adaptation of the tethered mitral valve: insights into a compensatory mechanism for functional mitral regurgitation. Circulation. 120(4):334-342.

Feinberg MS, Schwammenthal E, Shlizerman L, et al. 2000. Prognostic significance of mild mitral regurgitation by color Doppler echocardiography in acute myocardial infarction. The American journal of cardiology. 86(9):903-907.

Goldstein D, Moskowitz AJ, Gelijns AC, et al. 2016. Two-Year Outcomes of Surgical Treatment of Severe Ischemic Mitral Regurgitation. The New England journal of medicine. 374(4):344-353.

Grigioni F, Enriquez-Sarano M, Zehr KJ, Bailey KR, Tajik AJ. 2001. Ischemic mitral regurgitation: long-term outcome and prognostic implications with quantitative Doppler assessment. Circulation. 103(13):1759-1764.

He S, Fontaine AA, Schwammenthal E, Yoganathan AP, Levine RA. 1997. Integrated mechanism for functional mitral regurgitation: leaflet restriction versus coapting force: in vitro studies. Circulation. 96(6):1826-1834.

Hsuan CF, Yu HY, Tseng WK, Lin LC, Hsu KL, Wu CC. 2013. Quantitation of the mitral tetrahedron in patients with ischemic heart disease using real-time three-dimensional echocardiography to evaluate the geometric determinants of ischemic mitral regurgitation. Clinical cardiology. 36(5):286-292.

Hung J, Solis J, Handschumacher MD, Guerrero JL, Levine RA. 2012. Persistence of mitral regurgitation following ring annuloplasty: is the papillary muscle outside or inside the ring? The Journal of heart valve disease. 21(2):218-224.

Ji Q, Zhao Y, Shen J, et al. 2019. Risk Factors for Moderate or More Residual Regurgitation in Patients with Moderate Chronic Ischemic Mitral Regurgitation Undergoing Surgical Revascularization Alone. Int Heart J. 60(6):1268-1275.

Kono T, Sabbah HN, Rosman H, Alam M, Jafri S, Goldstein S. 1992. Left ventricular shape is the primary determinant of functional mitral regurgitation in heart failure. Journal of the American College of Cardiology. 20(7):1594-1598.

Kuwahara E, Otsuji Y, Iguro Y, et al. 2006. Mechanism of recurrent/persistent ischemic/functional mitral regurgitation in the chronic phase after surgical annuloplasty: importance of augmented posterior leaflet tethering. Circulation. 114(1 Suppl):I529-534.

Kwan J, Shiota T, Agler DA, et al. 2003. Geometric differences of the mitral apparatus between ischemic and dilated cardiomyopathy with significant mitral regurgitation: real-time three-dimensional echocardiography study. Circulation. 107(8):1135-1140.

Lehmann KG, Francis CK, Dodge HT. 1992. Mitral regurgitation in early myocardial infarction. Incidence, clinical detection, and prognostic implications. TIMI Study Group. Annals of internal medicine. 117(1):10-17.

Magne J, Pibarot P, Dagenais F, Hachicha Z, Dumesnil JG, Senechal M. 2007. Preoperative posterior leaflet angle accurately predicts outcome after restrictive mitral valve annuloplasty for ischemic mitral regurgitation. Circulation. 115(6):782-791.

McGee EC, Gillinov AM, Blackstone EH, et al. 2004. Recurrent mitral regurgitation after annuloplasty for functional ischemic mitral regurgitation. The Journal of thoracic and cardiovascular surgery. 128(6):916-924.

Meris A, Amigoni M, Verma A, et al. 2012. Mechanisms and predictors of mitral regurgitation after high-risk myocardial infarction. Journal of the American Society of Echocardiography : official publication of the American Society of Echocardiography. 25(5):535-542.

Michler RE, Smith PK, Parides MK, et al. 2016. Two-Year Outcomes of Surgical Treatment of Moderate Ischemic Mitral Regurgitation. The New England journal of medicine. 374(20):1932-1941.

Nesta F, Otsuji Y, Handschumacher MD, et al. 2003. Leaflet concavity: a rapid visual clue to the presence and mechanism of functional mitral regurgitation. Journal of the American Society of Echocardiography : official publication of the American Society of Echocardiography. 16(12):1301-1308.

Nishimura RA, Otto CM, Bonow RO, et al. 2017. 2017 AHA/ACC Focused Update of the 2014 AHA/ACC Guideline for the Management of Patients With Valvular Heart Disease: A Report of the American College of Cardiology/American Heart Association Task Force on Clinical Practice Guidelines. Circulation. 135(25):e1159-e1195.

Otsuji Y, Handschumacher MD, Schwammenthal E, et al. 1997. Insights from three-dimensional echocardiography into the mechanism of functional mitral regurgitation: direct in vivo demonstration of altered leaflet tethering geometry. Circulation. 96(6):1999-2008.

Otsuji Y, Kumanohoso T, Yoshifuku S, et al. 2002. Isolated annular dilation does not usually cause important functional mitral regurgitation: comparison between patients with lone atrial fibrillation and those with idiopathic or ischemic cardiomyopathy. Journal of the American College of Cardiology. 39(10):1651-1656.

Penicka M, Linkova H, Lang O, et al. 2009. Predictors of improvement of unrepaired moderate ischemic mitral regurgitation in patients undergoing elective isolated coronary artery bypass graft surgery. Circulation. 120(15):1474-1481.

Sabbah HN, Kono T, Stein PD, Mancini GB, Goldstein S. 1992. Left ventricular shape changes during the course of evolving heart failure. The American journal of physiology. 263(1 Pt 2):H266-270.

Sabbah HN, Rosman H, Kono T, Alam M, Khaja F, Goldstein S. 1993. On the mechanism of functional mitral regurgitation. The American journal of cardiology. 72(14):1074-1076.

Salmasi MY, Harky A, Chowdhury MF, et al. 2018. Should the mitral valve be repaired for moderate ischemic mitral regurgitation at the time of revascularization surgery? J Card Surg. 33(7):374-384.

Smith PK, Puskas JD, Ascheim DD, et al. 2014. Surgical treatment of moderate ischemic mitral regurgitation. The New England journal of medicine. 371(23):2178-2188.

Tibayan FA, Rodriguez F, Zasio MK, et al. 2003. Geometric distortions of the mitral valvular-ventricular complex in chronic ischemic mitral regurgitation. Circulation. 108 Suppl 1:Ii116-121.Zeng X, Nunes MC, Dent J, et al. 2014. Asymmetric versus symmetric tethering patterns in ischemic mitral regurgitation: geometric differences from three-dimensional transesophageal echocardiography. Journal of the American Society of Echocardiography: official publication of the American Society of Echocardiography. 27(4):367-375. 